\title{
Use of Lipid-Lowering Drugs and Associated Outcomes According to Health State Profiles in Hospitalized Older Patients
}

\author{
Carlotta Franchi (D) \\ Giulia Lancellotti ${ }^{2}$ \\ Marco Bertolotti ${ }^{2}$ \\ Simona Di Salvatore ${ }^{2}$ \\ Alessandro Nobili' \\ Pier Mannuccio Mannucci $\left(\mathbb{D}^{3}\right.$ \\ Chiara Mussi ${ }^{2} *$ \\ Ilaria Ardoino',*

\section{On Behalf of the REPOSI (REgistro POliterapie SIMI, Società Italiana di Medicina Interna) Study Group} \\ 'Department of Neuroscience, Istituto di \\ Ricerche Farmacologiche Mario Negri \\ IRCCS, Milano, Italy; ${ }^{2}$ Division of \\ Geriatrics, Department of Biomedical, \\ metabolic and Neural Sciences and \\ Center for Gerontological Evaluation and \\ Research, Università di Modena e Reggio \\ Emilia, Modena, Italy; ${ }^{3}$ Scientific \\ Direction, Fondazione IRCCS Ca' Granda \\ Ospedale Maggiore Policlinico, Milano, \\ Italy
}

*These authors contributed equally to this work
Correspondence: Carlotta Franchi Unit of Pharmacoepidemiological Research in Older People, Istituto di Ricerche Farmacologiche Mario Negri IRCCS, Via Mario Negri 2, Milano, 20I56, Italy

Tel +3902 390I4580

Fax +390239001916

Email carlotta.franchi@marionegri.it
Objective: To assess how lipid-lowering drugs (LLDs) are administered in the hospitalized patients aged 65 and older and their association with clinical outcomes according to their health-related profiles.

Design: This is a retrospective study based on data from REPOSI (REgistro POliterapie SIMI - Italian Society of Internal Medicine) register, an Italian network of internal medicine hospital wards.

Setting and Participants: A total of 4642 patients with a mean age of 79 years enrolled between 2010 and 2018.

Methods: Socio-demographic characteristics, functional abilities, cognitive skills, laboratory parameters and comorbidities were used to investigate the health state profiles by using multiple correspondence analysis and clustering. Logistic regression was used to assess whether LLD prescription was associated with patients' health state profiles and with shortterm mortality.

Results: Four clusters of patients were identified according to their health state: two of them (Cluster III and IV) were the epitome of frailty conditions with poor short-term outcomes, whereas the others included healthier patients. The average prevalence of LLD use was $27.6 \%$. The lowest prevalence was found among the healthier patients in Cluster I and among the oldest frail patients with severe functional and cognitive impairment in Cluster IV. The highest prevalence was among multimorbid patients in Cluster III $(\mathrm{OR}=4.50,95 \% \mathrm{CI}=3.76-5.38)$ characterized by a high cardiovascular risk. Being prescribed with LLDs was associated with a lower 3-month mortality, even after adjusting for cluster assignment $(\mathrm{OR}=0.59 ; 95 \% \mathrm{CI}=0.44-0.80)$.

Conclusion: The prevalence of LLD prescription was low and in overall agreement with guideline recommendations and with respect to patients' health state profiles.

Keywords: statins, health state profile, multimorbidity, polypharmacy

\section{Introduction}

Population ageing is a global phenomenon, ${ }^{1}$ with strong implications on the economic, social and healthcare systems. Ageing is associated with loss of homeostasis, decrease in physiological reserves and of the functional capacity to adapt to internal and external stressors, thereby leading to an increased vulnerability to disease and ultimately to frailty.

Cardiovascular events are the leading cause of morbidity and mortality in adults aged 65 and older, with coronary heart disease and stroke accounting for $60 \%$ of deaths in the oldest old. ${ }^{2}$ Among the most important risk factors for cardiovascular 
disease, there are lifestyle and smoking habits, hypertension, diabetes and hypercholesterolemia. Besides modifying inadequate lifestyles and diets, drugs may help to prevent cardiovascular diseases, especially in older populations. $^{3,4}$

Lipid-lowering drugs (LLDs), particularly statins, reduce mortality and atherothrombotic events, especially among high-risk patients and/or those for secondary prevention in the younger as well as in the older populations. ${ }^{4}$ Information from international guidelines is relatively scarce, mainly due to the limited evidence deriving from clinical trials in reducing atherothrombotic cardiovascular events. In particular, indication for treatment is controversial in primary prevention above the age of 75 years. ${ }^{4-8}$ In these patients, the management of hypercholesterolemia still represents a challenge, due to the high prevalence of comorbidities and polypharmacy that requires a comprehensive geriatric assessment of the complex health state of this increasing population. ${ }^{9}$

Health state assessment is a critical issue in the older people, and its evaluation is often problematic. ${ }^{9}$ The identification of health state profiles associated with different clinical outcomes was thought to provide a better practical support to address in older population the appropriate choice of pharmacological therapy.

With this background, we used a novel approach in order to investigate 1) the use of lipid-lowering drugs (LLDs) in relation to the patient health state and 2) the association of LLDs use to short-term mortality in a large cohort of older patients acutely hospitalized in Internal Medicine or Geriatric wards of the REPOSI (REgistro POliterapie SIMI) register.

\section{Methods}

\section{Setting}

The REPOSI register is a collaborative project promoted by the Italian Society of Internal Medicine (SIMI), the Fondazione IRCCS Cà Granda Ospedale Maggiore Policlinico and the Istituto di Ricerche Farmacologiche Mario Negri IRCCS in Milan, that is currently involving approximately 70 internal medicine and geriatrics hospital wards throughout Italy. The main purpose of the register is to investigate multimorbidity and polypharmacy, and their clinical-epidemiological correlates in a population of older patients hospitalized for any cause.

The REPOSI register enrols patients aged 65 years or older consecutively admitted to the participating wards during four index weeks (one per each season) per year. Data were initially obtained every two years (in 2008, 2010, 2012 and 2014) and yearly from 2016.

A standardized case report form (CRF) must be compiled for all admitted patients including socio-demographic characteristics, laboratory parameters, main comorbidities (Cumulative Illness Rating Scale - CIRS), ${ }^{10}$ ability in activity of daily life (Barthel Index - BI), ${ }^{11}$ cognitive skills (Short Blessed Test $-\mathrm{SBT})^{12}$ and the drugs prescribed at hospital admission, during hospitalization and at discharge. Data on mortality and hospital readmission were collected from 2012 onward by means of a telephone interview done 3 months after hospital discharge. More details are provided elsewhere. ${ }^{13,14}$ The study was conducted according to Good Clinical Practice and the Declaration of Helsinki, ${ }^{15}$ and was approved by the Ethical Committee of the IRCCS Ca' Granda Maggiore Policlinico Hospital Foundation of Milan and by the Ethics Committees of the participating centers. All patients provided signed informed consent.

All medical conditions were codified according to the International Classification of Diseases - Ninth Revision (ICD-9-CM) and all drugs according to the Anatomic Therapeutic Chemical Classification (ATC).

\section{Statistical Analysis}

Identification of Health-Related Profiles

In order to assess the health state profile, we used a previously described approach ${ }^{16}$ that integrates the Multiple Correspondence Analysis (MCA) and then the Hierarchical Clustering Analysis (HCA).

MCA aimed to discover relationships among several categorical variables. Continuous variables were also accommodated by splitting them into categories. MCA allows to reduce, with the least possible loss of information, a large number of correlated variables into few independent variables called factorial axis, whose values and interpretations are based on the categories of the original variables and may help to quantify unmeasurable phenomena such as the health state. ${ }^{17}$ Results of MCA can be graphically represented in a low dimensional space (eg the plane identified by the factorial axes). Categories of the original variables and/or individuals are represented on the plane as points with specific coordinates on each axis. Although the distance between points has no easy interpretation, the distances between points of categories of different variables may provide an approximate description of how different categories tend to be present together in the same individuals. 
HCA was then performed on the MCA scores of the obtained factorial axis in order to classify patients into homogeneous subsets based on their health state. ${ }^{18}$ The stability of the identified clusters was investigated by means of resampling. To this aim, 1000 bootstrap resamples with replacement were randomly generated from the original data. Each participant was then assigned to the cluster in which it was most frequently classified. ${ }^{19}$

To perform MCA (Supplementary Materials), we started from a large set of variables available in the REPOSI register and expected to be related to an unhealthy condition. Comorbidities with very low prevalence or with a negligible contribution to the structure of the data (ie to the explanation of the factorial axes) were discarded.

The variables finally included in the MCA analysis were:

- Sociodemographic, anthropometric and lifestyle data: sex, age, body mass index (BMI), living condition, smoking and alcohol consumption.

- Medical history: previous hospitalizations within 6 months, total number of diagnoses and drug intake, presence of illnesses (hypertension, diabetes, heart failure, ischemic heart diseases, atrial fibrillation and other arrhythmias, peripheral arteriopathy, chronic obstructive pulmonary disease [COPD], chronic kidney disease [CKD], arthritis and other musculoskeletal diseases, osteoporosis including fractures and prosthesis, stroke and Parkinson's disease).

- Performance in activities of daily living as measured by the BI.

- Cognition according to the SBT.

- Clinical and laboratory parameters: haemoglobin, glucose and estimated creatinine clearance. ${ }^{20}$

The diagnosis of dyslipidaemias and prescription of LLDs were not included in the MCA analysis meant to identify the clusters.

The original variables included in the MCA were then tabulated within the clusters to which the patients were allocated.

The association between cluster allocation and inhospital and 3-month mortality was assessed via logistic regression models.

\section{Use of LLDs and Association with Clinical Outcomes According to Patients' Health State Profiles}

The associations among diagnosis of dyslipidemia (ICD9: 272.*) and LLD use with the clusters identified were assessed via multinomial regression models, while the association between the LLD use with the short-term outcomes (in-hospital and 3-month mortality) via logistic regression. The results of logistic regression were reported using Odd Ratios (OR) and 95\% confidence interval $(95 \% \mathrm{CI})$.

LLDs (ATC: C10*) included both single drug (ie statins, fibrates, bile acid sequestrants and other LLDs) and drug combinations.

In order to assess in depth the proper use of LLDs among clusters, we also investigated situations in which statin use was not advisable, such as when they are involved in potential drug-drug interactions (DDI) or when they are used in pre-existing chronic conditions. Thus, we looked for the co-prescription among the most frequently prescribed statins (ie, atorvastatin and simvastatin) and calcium channel blockers, in particular: amlodipine (ATC: C08CA01), verapamil (C08DA01) and dilitiazem (C08DB01); and among simvastatin and amiodarone (C01BD01), ticagrelor (B01AC24) and dabigatran (B01AE07). Even the co-prescriptions of atorvastatin or rosuvastatin and fibrates were assessed. ${ }^{21,22}$

Moreover, because the use of statins is debated in patients with acute and/or decompensated liver diseases, this condition was also investigated. ${ }^{4}$

Statistical analysis was performed using SAS version 9.4 software (SAS Institute Inc., Cary, NC, USA) and $\mathrm{R}$ version 3.5 program.

\section{Results}

\section{Health-Related Profiles}

Overall, 6207 patients enrolled in 116 hospital wards participating in the REPOSI from 2010 onward were eligible for the study. The sample was well balanced between males (48.8\%) and females (51.2) and mean (std. dev.) age was 79 (7.5) years. In all, 4642 patients assessable for the variables of interest were used for the identification of the health state profiles by MCA (Figure 1).

According to the results of the MCA analysis, three factorial axes were retained that explained $70.5 \%$ of the total variance. The resulting figures showing the categories and the individuals according to cluster assignment projected on the plane identified by the factorial axes were depicted in Figures $\underline{\mathrm{S} 1}$ and $\underline{\mathrm{S}}$, along with a detailed explanation.

Four clusters were identified: two of them featured a broadly healthy profile, and the remaining two featured a state of frailty. Patients' characteristics according to their 


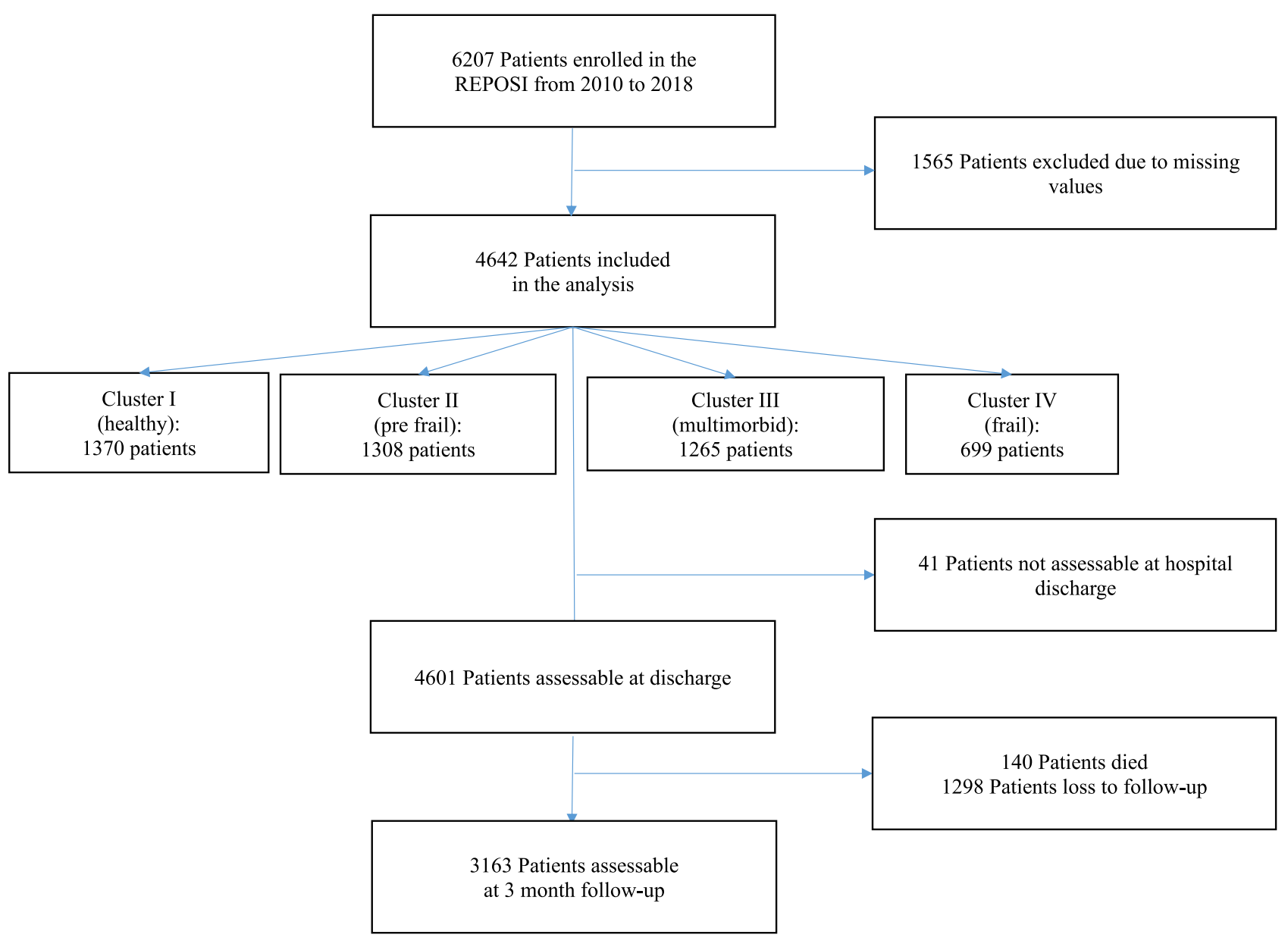

Figure I Flow-chart of the study.

health state profiles are reported in Table 1. Cluster I features healthy patients, mostly males $(68.8 \%)$, younger old (51.2\% patients aged less than 75 years), with an overall low prevalence of chronic conditions and negligible functional and cognitive declines. Cluster II features pre-frail patients, mostly women (93.4\%), mainly aged 75-85 years and with a healthy lifestyle. Despite their initial physiologic losses that mainly involved the metabolic (near $46 \%$ overweight or obese, $87.8 \%$ with hypertension and $27 \%$ with diabetes) and musculoskeletal systems (with high prevalence of osteoporosis and arthritis), nor functional defects nor cognitive impairments were evident in them. Cluster III features the multimorbid patients, mainly males ( $82.4 \%$ ), aged $75-85$ years, former smokers, on polypharmacy, with a high prevalence of diseases mainly related to the cardiovascular systems (87.8\% hypertension, $33.4 \%$ heart failure and $60 \%$ ischemic heart disease), renal failure (45.2\%) and diabetes $(50.6 \%)$. In them, functional disabilities and losses in specific capabilities are becoming evident because nearly
$25 \%$ of them had at least a moderate functional dependence. Cluster IV features frail patients, with the highest prevalence of oldest old, mainly females (70.2\%) affected by cerebrovascular ( $37.6 \%$ patients had previous stroke) and musculoskeletal diseases and severe cognitive impairment and functional disability (64\%).

Of the 4642 patients assessed, 41 of them were not assessable at hospital discharge and 140 (3.0\%) died during hospitalization (Figure 1). Table 2 shows the results of the logistic regression model of the associations of health state profiles and statin use with outcomes. In particular, Cluster III and much more so Cluster IV were significantly associated $(p<0.0001)$ with higher in-hospital mortality.

Among the 4461 patients assessable at hospital discharge, 3136 were also assessable at 3-month follow-up and 314 died (Figure 1). Cluster III and much more so Cluster IV were again associated $(p<0.0001)$ with shortterm mortality. On the other hand, female patients included in Cluster II showed lower 3-month mortality than those, almost men, included in Cluster I $(\mathrm{p}=0.01)$. 
Table I Patients' Characteristics at Hospital Admission According to the Health State Profile Identified

\begin{tabular}{|c|c|c|c|c|}
\hline Variables & $\begin{array}{l}\text { Cluster I } \\
N(\%) \\
1370\end{array}$ & $\begin{array}{l}\text { Cluster II } \\
N(\%) \\
1308\end{array}$ & $\begin{array}{l}\text { Cluster III } \\
\mathrm{N}(\%) \\
\text { I } 265\end{array}$ & $\begin{array}{l}\text { Cluster IV } \\
\mathrm{N}(\%) \\
699\end{array}$ \\
\hline \multicolumn{5}{|l|}{ Gender } \\
\hline Male & $942(68.8)$ & $87(6.6)$ & $1042(82.4)$ & $208(29.8)$ \\
\hline Famale & $428(31.2)$ & $|22|(93.4)$ & $223(17.6)$ & $491(70.2)$ \\
\hline \multicolumn{5}{|l|}{ Age (years) } \\
\hline Young old (60-74) & $702(5 \mid .2)$ & $342(26.1)$ & $361(28.5)$ & $39(5.6)$ \\
\hline Middle old (75-84) & $537(39.2)$ & $660(50.5)$ & $669(52.9)$ & $220(31.5)$ \\
\hline Oldest old $(85+)$ & |3| (9.6) & $306(23.4)$ & $235(18.6)$ & $440(62.9)$ \\
\hline \multicolumn{5}{|l|}{ Body mass index (BMI) * } \\
\hline Underweight & $425(31.0)$ & $324(24.8)$ & $254(20.1)$ & $310(44.4)$ \\
\hline Normal weight & $570(4 I .6)$ & $385(29.4)$ & $470(37.1)$ & $212(30.3)$ \\
\hline Overweight & $240(17.5)$ & $262(20.0)$ & $278(22.0)$ & 95 (13.6) \\
\hline Obese & $135(9.9)$ & $337(25.8)$ & $263(20.8)$ & $82(11.7)$ \\
\hline \multicolumn{5}{|l|}{ Living condition } \\
\hline Alone & $292(21.3)$ & $512(39.1)$ & $201(15.9)$ & $85(12.2)$ \\
\hline With relatives & $970(70.8)$ & $699(53.5)$ & $946(74.8)$ & $372(53.2)$ \\
\hline Caregiver & $43(3.1)$ & $58(4.4)$ & $55(4.4)$ & $146(20.9)$ \\
\hline Nursing home & $65(4.8)$ & $39(3.0)$ & $63(5.0)$ & $96(13.7)$ \\
\hline \multicolumn{5}{|l|}{ Alcohol } \\
\hline Never & $469(34.2)$ & $997(76.2)$ & $373(29.5)$ & $443(63.4)$ \\
\hline Ex drinker & $92(6.7)$ & $19(1.5)$ & $134(10.6)$ & 41 (5.9) \\
\hline Drinker & $809(59.1)$ & $292(22.3)$ & $758(59.9)$ & $215(30.7)$ \\
\hline \multicolumn{5}{|l|}{ Smoke } \\
\hline Never & $513(37.5)$ & II 47(87.7) & $301(23.8)$ & 501 (7I.7) \\
\hline Ex-smoker & $636(46.4)$ & $127(9.7)$ & $852(67.3)$ & $174(24.9)$ \\
\hline Smoker & $221(16.1)$ & $34(2.6)$ & $112(8.9)$ & $24(3.4)$ \\
\hline \multicolumn{5}{|l|}{ Barthel index $(\mathrm{BI})^{\dagger}$} \\
\hline No or negligible dependence & $1080(78.8)$ & $724(55.4)$ & $667(52.7)$ & $50(7.2)$ \\
\hline Mild dependence & $177(12.9)$ & $347(26.5)$ & $287(22.7)$ & $72(10.3)$ \\
\hline Moderate & $69(5.0)$ & $160(12.2)$ & $195(15.4)$ & $132(18.9)$ \\
\hline Severe dependence & $23(1.7)$ & $54(4.1)$ & $79(6.3)$ & $168(24.0)$ \\
\hline Total dependence & $21(1.6)$ & $23(1.8)$ & $37(2.9)$ & 277 (39.6) \\
\hline \multicolumn{5}{|l|}{ Short blessed test (SBT) } \\
\hline Normal $(0-4)$ & $783(57.2)$ & $522(39.9)$ & $494(39.1)$ & $47(6.7)$ \\
\hline Possible cog imp (5-9) & $226(16.5)$ & $280(21.4)$ & $267(21.1)$ & $37(5.3)$ \\
\hline Moderate cog imp (10-19) & $314(22.9)$ & $455(34.8)$ & $45 I(35.6)$ & $168(24.0)$ \\
\hline Severe cog imp (20-28) & $47(3.4)$ & 51 (3.9) & $53(4.2)$ & $447(64.0)$ \\
\hline \multicolumn{5}{|l|}{ Hemoglobin $¥$} \\
\hline No anemia & $727(53.1)$ & $640(48.9)$ & $386(30.5)$ & $248(35.5)$ \\
\hline Mild anemia & $322(23.5)$ & $220(16.8)$ & $436(34.5)$ & $140(20.0)$ \\
\hline Moderate anemia & $238(17.4)$ & 391 (29.9) & $373(29.5)$ & $260(37.2)$ \\
\hline Severe anemia & $83(6.0)$ & $57(4.4)$ & $70(5.5)$ & $51(7.3)$ \\
\hline \multicolumn{5}{|l|}{ Glucose $^{\S}$} \\
\hline Normal & 601 (43.9) & $501(38.3)$ & $360(28.5)$ & $264(37.8)$ \\
\hline High & $474(34.6)$ & $357(27.3)$ & $311(24.6)$ & $210(30.0)$ \\
\hline Very high & $295(21.5)$ & $450(34.4)$ & $594(46.9)$ & 225 (32.2) \\
\hline
\end{tabular}

(Continued) 
Table I (Continued).

\begin{tabular}{|c|c|c|c|c|}
\hline Variables & $\begin{array}{l}\text { Cluster I } \\
N(\%) \\
1370\end{array}$ & $\begin{array}{l}\text { Cluster II } \\
N(\%) \\
1308\end{array}$ & $\begin{array}{l}\text { Cluster III } \\
N(\%) \\
1265\end{array}$ & $\begin{array}{l}\text { Cluster IV } \\
\mathrm{N}(\%) \\
699\end{array}$ \\
\hline \multicolumn{5}{|l|}{ Creatinine clearance } \\
\hline Stage $\mid>90$ & $301(22.0)$ & $89(6.8)$ & $54(4.3)$ & $32(4.6)$ \\
\hline Stage II $\leq 90$ & 725 (52.9) & $567(43.4)$ & 389 (30.7) & $227(32.5)$ \\
\hline Stage III $\leq 60$ & $311(22.7)$ & $526(40.2)$ & $52 \mid(4 \mid .2)$ & $297(42.5)$ \\
\hline Stage IV $\leq 30$ & $22(1.6)$ & $97(7.4)$ & 239 (18.9) & $99(14.1)$ \\
\hline Stage $V \leq 15$ & II (0.8) & $29(2.2)$ & $62(4.9)$ & $44(6.3)$ \\
\hline \multicolumn{5}{|l|}{ Number of drugs } \\
\hline $0-1$ & $318(23.2)$ & $30(2.3)$ & $3(0.2)$ & $35(5.0)$ \\
\hline $2-4$ & $680(49.6)$ & $475(36.3)$ & II4 (9.0) & $195(27.9)$ \\
\hline $5-9$ & $366(26.7)$ & $733(56.0)$ & $811(64.1)$ & $406(58.1)$ \\
\hline$>10$ & $6(0.5)$ & $70(5.4)$ & $337(26.7)$ & $63(9.0)$ \\
\hline \multicolumn{5}{|l|}{ Number of diagnosis } \\
\hline Healthy $(0-1)$ & $161(11.8)$ & $17(1.3)$ & $0(0.0)$ & $9(1.3)$ \\
\hline Morbid (2-4) & $811(59.2)$ & $435(33.3)$ & $63(5.0)$ & $110(15.7)$ \\
\hline Multimorbid $(>5)$ & $398(29.0)$ & $856(65.4)$ & $1202(95.0)$ & $580(83.0)$ \\
\hline Previous hospitalization & $289(21.1)$ & $370(28.3)$ & $629(49.7)$ & $276(39.5)$ \\
\hline \multicolumn{5}{|l|}{ Main Diagnosis: } \\
\hline Hypertension & $74 I(54.1)$ & $1149(87.8)$ & $1138(90.0)$ & $559(80.0)$ \\
\hline CKD $\|$ & $35(2.6)$ & $135(10.3)$ & $572(45.2)$ & $164(23.5)$ \\
\hline Diabetes & $161(11.8)$ & $354(27.1)$ & $640(50.6)$ & 124 (17.7) \\
\hline COPD ** & $194(14.2)$ & $135(10.3)$ & $494(39.0)$ & $168(24.0)$ \\
\hline Heart failure & 51 (3.7) & $183(14.0)$ & $422(33.4)$ & $168(24.0)$ \\
\hline Parkinson's & $18(1.3)$ & $19(1.5)$ & $32(2.5)$ & $86(12.3)$ \\
\hline Osteoporosis & $72(5.3)$ & $203(15.5)$ & $73(5.8)$ & $149(21.3)$ \\
\hline Ischemic heart disease & $146(10.7)$ & $209(16.0)$ & $631(49.9)$ & $162(23.2)$ \\
\hline Arthritis & $144(10.5)$ & $430(32.9)$ & $195(15.4)$ & $148(2 \mid .2)$ \\
\hline Stroke/TIA & $159(11.6)$ & $164(12.5)$ & $252(19.9)$ & $263(37.6)$ \\
\hline Atrial fibrillation & $156(11.4)$ & $384(29.4)$ & 479 (37.9) & $236(33.8)$ \\
\hline $\begin{array}{l}\text { Peripheral arterial } \\
\text { disease }\end{array}$ & $68(5.0)$ & $97(7.4)$ & $322(25.5)$ & $95(13.6)$ \\
\hline Dyslipidemia & 91 (6.6) & $145(11.1)$ & $164(13.0)$ & $44(6.3)$ \\
\hline Statin user & $230(16.8)$ & $335(25.6)$ & $602(47.6)$ & $115(16.5)$ \\
\hline
\end{tabular}

Notes:*BMI: Underweight (<23), Normal weight ( $\geq 23$ and $<27)$, Overweight ( $\geq 27$ and $<30)$, Obese ( $\geq 30) ;{ }^{\dagger}$ Barthel Index: No Dependence (9I-I00), Mild Dependence (75-90), Moderate Dependence (50-74), Severe Dependence (25-49), Total Dependence (0-24); ${ }^{\ddagger}$ Hemoglobin: No anemia (male: $\geq 13 \mathrm{~g} / \mathrm{dL}$, female: $\left.\geq 12 \mathrm{~g} / \mathrm{dL}\right)$, mild anemia (male: $\geq 1 \mathrm{~g} / \mathrm{dL}$ and $<13 \mathrm{~g} / \mathrm{dL}$, female: $\geq 1 \mathrm{~g} / \mathrm{dL}$ and $<12 \mathrm{~g} / \mathrm{dL}$ ), moderate anemia (male and female: $\geq 8 \mathrm{~g} / \mathrm{dL}$ and $<1 / \mathrm{g} / \mathrm{dL}$ ), severe anemia (male and female: $<8 \mathrm{~g} / \mathrm{dL}$ ); ${ }^{\S}$ Glucose: Normal ( $<100 \mathrm{mg} / \mathrm{dL}$ ), High ( $\geq 100 \mathrm{mg} / \mathrm{dL}$ and $<125 \mathrm{mg} / \mathrm{dL}$ ), Very High $(\geq 125 \mathrm{mg} / \mathrm{dL})$; ICD-9-CM code for assessing main diagnosis: Hypertension: 401 (CIRS item 2); " CKD: Chronic Kidney Disease: 585; Diabetes: 250; ** COPD: Chronic Obstructive Pulmonary Disease: 49I; Heart failure: 402.*I, 428; Parkinson's: 322; Osteoporosis (including Fractures and prosthesis): 733, 829, V436; Ischemic heart disease: 410-414; Arthritis (and other rheumatic diseases): 710-729; Stroke: 430-438; Atrial fibrillation (and other arrhythmias): 427; Peripheral arterial disease: 440-44I; Dyslipidemia: 272.

\section{Use of LLDs and Association with Clinical Outcomes According to Health State} Profiles

Diagnosis of dyslipidemias and prescription of LLDs were found to be associated with the health state profiles (Table 3). An increased incidence of dyslipidemia was found in Clusters II $(\mathrm{OR}=1.75,95 \% \mathrm{CI}=1.33-2.30)$ and III $(\mathrm{OR}=2.10,95 \% \mathrm{CI}=1.60-2.74)$.

All in all, $1282(27.6 \%)$ patients were prescribed with at least one LLD: 1198 patients taking only one, 83 patients taking two and one patient (in Cluster III) taking 3 different active substances. Although information was partly lacking 
Table 2 Results of Logistic Regression Model (or, 95\% Cl) to Assess the Impact of the Health State Profile and Statin Use, Adjusted for Health State Profile, on Main Clinical Outcomes

\begin{tabular}{|c|c|c|c|c|c|c|}
\hline & \multicolumn{6}{|c|}{ Main Outcomes } \\
\hline & \multicolumn{3}{|c|}{ In Hospital Mortality* } & \multicolumn{3}{|c|}{ 3-Month Mortality** } \\
\hline \multirow[b]{2}{*}{ Cluster I } & $\mathbf{N}$ & \multicolumn{2}{|c|}{ OR (95\% CI) } & \multirow{2}{*}{$\begin{array}{c}\mathbf{N} \\
76\end{array}$} & \multicolumn{2}{|c|}{ OR (95\% Cl) } \\
\hline & 18 & Ref. & Ref. & & Ref. & Ref \\
\hline Cluster II & 14 & $0.81(0.40-1.63)$ & $0.83(0.4 \mathrm{I}-\mathrm{I} .67)$ & 50 & $0.64(0.44-0.92)$ & $0.66(0.46-0.96)$ \\
\hline Cluster III & 42 & $2.56(1.47-4.47)$ & $2.79(1.58-4.93)$ & 107 & $1.48(1.09-2.02)$ & I.7I (1.24-2.36) \\
\hline Cluster IV & 66 & $7.78(4.58-13.21)$ & $7.78(4.58-13.21)$ & 81 & $2.45(1.75-3.43)$ & $2.46(1.76-3.45)$ \\
\hline Statin use & & - & $0.75(0.49-1.14)$ & & - & $0.59(0.44-0-80)$ \\
\hline
\end{tabular}

Notes: *The model was fitted using $460 \mathrm{l}$ observation; **The model was fitted using 3136 observation.

Abbreviations: OR, odds ratio; $95 \% \mathrm{Cl}, 95 \%$ confidence interval.

Table 3 Results of Logistic Regression Model (or $(95 \% \mathrm{Cl})$ ) to Assess the Association Among Diagnosis of Dyslipidemia $(A)$ and Statin Prescription (B) with Cluster Assignment

\begin{tabular}{|l|c|r|r|r|}
\hline & \multicolumn{2}{|c|}{ (A) - Dyslipidemia } & \multicolumn{2}{c|}{ (B) - Statin User } \\
\hline & N & OR (95\% CI) & N & OR (95\% CI) \\
\hline Cluster I & 91 & Ref. & 230 & Ref. \\
\hline Cluster II & 145 & $1.75(I .33-2.30)$ & 335 & $1.70(I .4 I-2.06)$ \\
\hline Cluster III & 164 & $2.09(1.60-2.78)$ & 602 & $4.50(3.76-5.38)$ \\
\hline Cluster IV & 66 & $0.95(0.65-1.37)$ & 115 & $0.98(0.76-1.25)$ \\
\hline
\end{tabular}

Abbreviations: OR, odds ratio; $95 \% \mathrm{Cl}, 95 \%$ confidence interval.

due to missing data, almost all patients (eg 1054-91.5\% - out of 1152 patients) has been prescribed with LLDs for at least 6 months before hospital admission.

The most prescribed drugs (overall and for each cluster) were statins: firstly, atorvastatin $(\mathrm{N}=553)$, which accounts for $40.5 \%$ of all prescriptions, followed by simvastatin $(\mathrm{N}=374,27.4 \%)$ and rosuvastatin $(\mathrm{N}=189$, $13.8 \%)$. Only few patients $(\mathrm{N}=43)$ were prescribed with the combination of simvastatin and ezetimibe (Figure 2). No significant differences were observed among clusters, but with respect to the overall population atorvastatin was more prescribed in Clusters III and IV, simvastatin was more prescribed in cluster I and IV and less prescribed in Cluster III, while all the other LLDs were less prescribed in cluster IV.

The most prescribed patients were those in Cluster III, who were in average 75 years old and affected by cardiovascular diseases $(\mathrm{OR}=4.50,95 \% \mathrm{CI}=3.76-5.38)$. Even females in Cluster II received statins more often than patients included in Cluster I and III $(\mathrm{OR}=1.70,95 \% \mathrm{CI}$ $=1.41-2.06$ ).

All in all, 202 patients out of $1282(15.8 \%)$ had at least one DDI, in particular among the most prescribed statins with calcium channel blockers $(\mathrm{N}=169)$, with an increasing prevalence in the multimorbid patients in Cluster III. The incidence of liver disease among the prescribed patients was lower $(5.3 \%)$ than in the overall population (near the 9.0\%), with less cases in the Cluster I.

Being prescribed with lipid lowering drugs was associated with a lower 3-month mortality, even after adjusting for cluster assignment $(\mathrm{OR}=0.59 ; 95 \% \mathrm{CI}=0.44-0.80)$ (Table 2).

\section{Discussion}

We chose to analyse the data stemming from the REPOSI register of hospitalized older patients in order to identify the main features defining clusters of patients based on their health state profiles, with the ultimate goal to evaluate whether or not there was a relationship between the prescription of lipid-lowering drugs and the health state phenotypes identified. The analysis identified four different clusters of patients, two of them (III and IV) being characterized by a frailty profile, associated with multimorbidities and disabilities. An overall low prevalence of statin prescription (slightly higher than 25\%) was found. Among the multimorbid patients of Cluster III with a high cardiovascular risk, the prevalence of statin use was the highest (nearly 50\%), whereas the lowest prevalence $(16.5 \%)$ was recorded among the frail patients of Cluster IV characterized by functional and cognitive impairments, poor nutritional state and poor clinical outcome. 


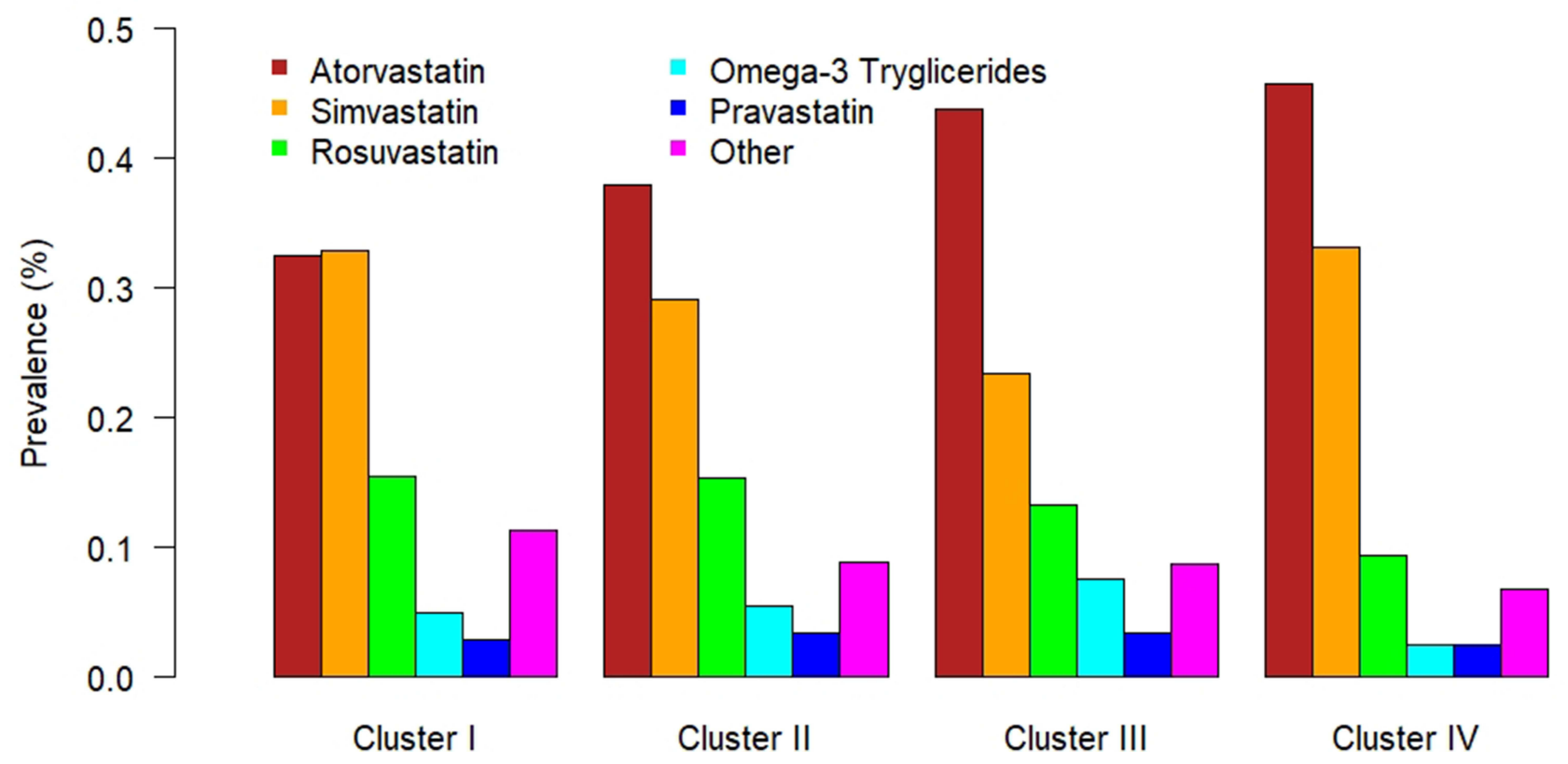

Figure 2 Barplot of the distribution of lipid lowering drugs according to cluster assignment.

The overall low prevalence of statin use in our population was not dissimilar to that reported in the worldwide adult population aged $40+{ }^{21}$ Despite their undisputed efficacy in the prevention and treatment of cardiovascular diseases, the poor use could be explained by the intolerance mainly associated with muscle symptoms and related adverse events. ${ }^{23}$ Moreover, new LLDs, such as bempedoic acid, a non-statin drug that targets cholesterol biosynthesis and provides promising results both alone or in combination with statins, ${ }^{21}$ might have offered a valid alternative in these patients, especially in those enrolled in the last runs of the REPOSI register. ${ }^{24}$

The highest prevalence of statin use found in the Cluster III of patients at high risk (around 50\%), is generally in line with data reported for secondary prevention in the Italian population, even if a direct age-matched comparison is not easy to achieve. The National Report on medicine use in Italy describes an overall use of LLDs of nearly $50 \%$ in all subjects with a diagnosis of dyslipidemia, with a prevalence of such diagnosis ranging from $33 \%$ to $41 \%$ in the over $65 .{ }^{25}$ Another report in the older patients on secondary cardiovascular prevention described a similar prevalence of statin use $(49 \%),{ }^{26}$ whereas data collected in high-risk subjects with a mean age of 71 showed a $53 \%$ prevalence of use. ${ }^{27}$

The management of cardiovascular risk in older adults still represents a critical challenge, owing to poor evidence and scanty guidelines for this population. ${ }^{28}$ A recent large meta-analysis confirmed a protective effect of statins in older patients, even if most of the studies considered were not specifically designed for this age group and the benefit was less evident in primary prevention above age $75 .^{29}$ Lipid-lowering drugs are considered effective and safe for primary prevention in the older as well as in younger patients with a high risk of cardiovascular disease and for secondary prevention in those with a history of cardiovascular events (Class I level A evidence according to the ESC guideline). ${ }^{4,29,30}$ On the other hand, the use of statins in primary prevention for moderate cardiovascular risk patients is much more debated and less evidence is available (Class IIb Level B). ${ }^{4,30} \mathrm{~A}$ similar approach is suggested by the recent AHA/ACC guidelines, ${ }^{8}$ whereas no evidence of benefit is available in patients aged 80 years or more with no previous coronary artery disease, peripheral vascular disease or cerebrovascular disease. ${ }^{28,30}$ The use of statins should be carefully evaluated and generally avoided in patients with a small body frame, multimorbidity and exposed to polypharmacy, ie, the epitome of the frailest older people. ${ }^{31}$ The decision to continue therapy in the oldest elderly may also be influenced by impaired physical and cognitive functions.

Furthermore, the assessment of prescription appropriateness may be especially challenging in hospitalized older patients with varied sociodemographic, clinical and pharmacological features, such as those participating in the 
REPOSI register. Thus, we choose to implement a methodological approach based on the identification of health state profiles with the goal to make a comprehensive assessment of these patients who needed more focused and personalized strategies.

With this background, the results of this study may be interpreted as indicating an overall use of statins among older hospitalized patients broadly in agreement with the recommendations of the guidelines. The prevalence of use of LLDs was $27.6 \%$ at hospital admission. This average prevalence was found in patients from Cluster II featuring healthy females presenting with musculoskeletal-related deficits and some early evidence of the metabolic syndrome (hypertension, diabetes, overweigh). Among them, the prevalence of dyslipidemia was higher than in the overall sample and similar to that observed among multimorbid patients from Cluster III (11\% vs 13\%), who presented a moderate to high cardiovascular risk. The highest prevalence of statin use (nearly 50\%) was observed in Cluster III that gathered patients in the context of secondary prevention of cardiovascular disease (heart failure, ischemic heart disease) or with a high cardiovascular risk (hypertension, diabetes, former smokers and alcohol drinker) even if they had little or no cognitive impairment and few functional limitations. There was a lower prevalence of statin use among patients from Clusters I and IV. Cluster I included mainly healthier males, younger old, with low to moderate cardiovascular risk, so that this low prevalence was expected and appropriate. On the other hand, patients from Cluster IV had the highest prevalence of previous stroke/TIA (37.6\%) and other conditions associated with a high cardiovascular risk, but they were also the oldest old with more severe cognitive impairment and loss of functional independence. On the whole, their health state profile apparently justifies the low prevalence of statin use, in agreement with guidelines and recommendations stemming from the literature, ${ }^{4-8,31,32}$ but in contrast with the findings of Borne et al, who observed an overuse of statins for primary prevention in the communitydwelling population aged 80 years or more. ${ }^{33}$ The observed relatively low prevalence of drug-drug interactions, albeit increasing in Cluster III further supports our concluding remark of an overall proper use of LLDs in this hospitalized population of older patients.

\section{Strengths and Limitations}

The main strength of this study is the large number of internal medicine and geriatric wards throughout Italy participating in the REPOSI register, which provide a representative and real-world sample of older hospitalized patients that reflect the overall prescribing habits of Italian clinicians. On the other hand, the real-world data collection in the frame of a register leads to a partial lack of collected data and to a huge number of missing data. In this context, the record linkage of data from different sources (eg health administrative database) might be an opportunity to improve the quality of the data collected in real-life studies.

The missing total cholesterol serum values in a large fraction of the sample, for example, prevented us from accurately assessing cardiovascular risk scores. Some diagnoses or specific conditions (such as malnutrition, sensorial deficit etcetera) might also be underreported. Finally, although the identification of health state phenotype helps to recognize patients' need according to different cardiovascular risk profiles, it did not allow us to evaluate the appropriateness of prescription at the individual level.

\section{Conclusion}

The decision whether or not a pharmacological treatment with lipid-lowering drugs should be implemented and/or continued in hospitalized older adults needs to be individually tailored to each patient. At the time of prescribing, consideration should be given to life expectancy, comorbidities, polypharmacy and increased risks of adverse reactions in the geriatric population, especially in the oldest old. ${ }^{34}$ Our approach used herewith in order to identify the health state profiles of older patients may also be useful both for the management and assessment of this and of other pharmacological treatments in older patients admitted to hospitals.

\section{Data Sharing Statement}

Not applicable.

\section{Acknowledgments}

REPOSI collaborators list:

Steering Committee: Pier Mannuccio Mannucci (Chair) (Fondazione IRCCS Cà Granda Ospedale Maggiore Policlinico, Milano), Alessandro Nobili (cochair) (Istituto di Ricerche Farmacologiche Mario Negri IRCCS, Milano), Antonello Pietrangelo (Presidente SIMI), Francesco Perticone (Direttore CRIS - SIMI), Giuseppe Licata (Socio d'onore SIMI), Francesco Violi (Policlinico Umberto I, Roma, Prima Clinica Medica), Gino Roberto 
Corazza (Reparto 11, IRCCS Policlinico San Matteo di Pavia, Pavia, Clinica Medica I), Salvatore Corrao (ARNAS Civico, Di Cristina, Benfratelli, DiBiMIS, Università di Palermo, Palermo), Alessandra Marengoni (Spedali Civili di Brescia, Brescia), Francesco Salerno (IRCCS Policlinico San Donato Milanese, Milano), Matteo Cesari (UO Geriatria, Università degli Studi di Milano), Mauro Tettamanti, Luca Pasina, Carlotta Franchi (Istituto di Ricerche Farmacologiche Mario Negri IRCCS, Milano).

Clinical data monitoring and revision: Carlotta Franchi, Laura Cortesi, Mauro Tettamanti, Gabriella Miglio (Istituto di Ricerche Farmacologiche Mario Negri IRCCS, Milano).

Database Management and Statistics: Mauro Tettamanti, Laura Cortesi, Ilaria Ardoino, Alessio Novella (Istituto di Ricerche Farmacologiche Mario Negri IRCCS, Milano).

\section{Investigators}

- Domenico Prisco, Elena Silvestri, Giacomo Emmi, Alessandra Bettiol, Irene Mattioli (Azienda Ospedaliero Universitaria Careggi Firenze, Medicina Interna Interdisciplinare);

- Gianni Biolo, Michela Zanetti, Giacomo Bartelloni (Azienda Sanitaria Universitaria Integrata di Trieste, Clinica Medica Generale e Terapia Medica);

- Massimo Vanoli, Giulia Grignani, Edoardo Alessandro Pulixi (Azienda Ospedaliera della Provincia di Lecco, Ospedale di Merate, Lecco, Medicina Interna);

- Graziana Lupattelli, Vanessa Bianconi, Riccardo Alcidi (Azienda Ospedaliera Santa Maria della Misericordia, Perugia, Medicina Interna);

- Domenico Girelli, Fabiana Busti, Giacomo Marchi (Azienda Ospedaliera Universitaria Integrata di Verona, Verona, Medicina Generale e Malattie Aterotrombotiche e Degenerative);

- Mario Barbagallo, Ligia Dominguez, Vincenza Beneduce, Federica Cacioppo (Azienda Ospedaliera Universitaria Policlinico Giaccone Policlinico di Palermo, Palermo, Unità Operativa di Geriatria e Lungodegenza);

- Salvatore Corrao, Giuseppe Natoli, Salvatore Mularo, Massimo Raspanti, (A.R.N.A.S. Civico, Di Cristina, Benfratelli, Palermo, UOC Medicina Interna ad Indirizzo Geriatrico-Riabilitativo);

- Marco Zoli, Maria Laura Matacena, Giuseppe Orio, Eleonora Magnolfi, Giovanni Serafini, Angelo Simili
(Azienda Ospedaliera Universitaria Policlinico S. Orsola-Malpighi, Bologna, Unità Operativa di Medicina Interna);

- Giuseppe Palasciano, Maria Ester Model, Carla Di Gennaro (Azienda Ospedaliero-Universitaria Consorziale Policlinico di Bari, Bari, Medicina Interna Ospedaliera "L. D'Agostino", Medicina Interna Universitaria “A. Murri”);

- Maria Domenica Cappellini, Giovanna Fabio, Margherita Migone De Amicis, Giacomo De Luca, Natalia Scaramellini (Fondazione IRCCS Cà Granda Ospedale Maggiore Policlinico, Milano, Unità Operativa Medicina Interna IA);

- Matteo Cesari, Paolo Dionigi Rossi, Sarah Damanti, Marta Clerici, Simona Leoni, Alessandra Danuta Di Mauro (Fondazione IRCCS Cà Granda Ospedale Maggiore Policlinico, Milano, Geriatria);

- Antonio Di Sabatino, Emanuela Miceli, Marco Vincenzo Lenti, Martina Pisati, Costanza Caccia Dominioni (IRCCS Policlinico San Matteo di Pavia, Pavia, Clinica Medica I, Reparto 11);

- Roberto Pontremoli, Valentina Beccati, Giulia Nobili, Giovanna Leoncini (IRCCS Azienda Ospedaliera Universitaria San Martino-IST di Genova, Genova, Clinica di Medicina Interna 2);

- Luigi Anastasio, Maria Carbone (Ospedale Civile Jazzolino di Vibo Valentia, Vibo Valentia, Medicina interna);

- Francesco Cipollone, Maria Teresa Guagnano, Ilaria Rossi (Ospedale Clinicizzato SS. Annunziata, Chieti, Clinica Medica);

- Gerardo Mancuso, Daniela Calipari, Mosè Bartone (Ospedale Giovanni Paolo II Lamezia Terme, Catanzaro, Unità Operativa Complessa Medicina Interna);

- Giuseppe Delitala, Maria Berria, Alessandro Delitala (Azienda ospedaliera-universitaria di Sassari, Clinica Medica);

- Maurizio Muscaritoli, Alessio Molfino, Enrico Petrillo, Antonella Giorgi, Christian Gracin (Policlinico Umberto I, Sapienza Università di Roma, Medicina Interna e Nutrizione Clinica Policlinico Umberto I);

- Giuseppe Zuccalà, Gabriella D'Aurizio (Policlinico Universitario A. Gemelli, Roma, Roma, Unità Operativa Complessa Medicina d'Urgenza e Pronto Soccorso)

- Giuseppe Romanelli, Alessandra Marengoni, Andrea Volpini, Daniela Lucente (Unità Operativa Complessa 
di Medicina I a indirizzo geriatrico, Spedali Civili, Montichiari (Brescia));

- Antonio Picardi, Umberto Vespasiani Gentilucci, Paolo Gallo (Università Campus Bio-Medico, Roma, Medicina Clinica-Epatologia);

- Giuseppe Bellelli, Maurizio Corsi, Cesare Antonucci, Chiara Sidoli, Giulia Principato (Università degli studi di Milano-Bicocca Ospedale S. Gerardo, Monza, Unità Operativa di Geriatria);

- Franco Arturi, Elena Succurro, Bruno Tassone, Federica Giofrè (Università degli Studi Magna Grecia, Policlinico Mater Domini, Catanzaro, Unità Operativa Complessa di Medicina Interna);

- Maria Grazia Serra, Maria Antonietta Bleve (Azienda Ospedaliera "Cardinale Panico" Tricase, Lecce, Unità Operativa Complessa Medicina);

- Antonio Brucato Teresa De Falco (ASST Fatebenefratelli - Sacco, Milano, Medicina Interna);

- Fabrizio Fabris, Irene Bertozzi, Giulia Bogoni, Maria Victoria Rabuini, Tancredi Prandini (Azienda Ospedaliera Università di Padova, Padova, Clinica Medica I);

- Roberto Manfredini, Fabio Fabbian, Benedetta Boari, Alfredo De Giorgi, Ruana Tiseo (Azienda Ospedaliera - Universitaria Sant'Anna, Ferrara, Unità Operativa Clinica Medica);

- Giuseppe Paolisso, Maria Rosaria Rizzo, Claudia Catalano (Azienda Ospedaliera Universitaria della Seconda Università degli Studi di Napoli, Napoli, VI Divisione di Medicina Interna e Malattie Nutrizionali dell'Invecchiamento);

- Claudio Borghi, Enrico Strocchi, Eugenia Ianniello, Mario Soldati, Silvia Schiavone, Alessio Bragagni (Azienda Ospedaliera Universitaria Policlinico S. OrsolaMalpighi, Bologna, Unità Operativa di Medicina Interna Borghi);

- Carlo Sabbà, Francesco Saverio Vella, Patrizia Suppressa, Giovanni Michele De Vincenzo, Alessio Comitangelo, Emanuele Amoruso, Carlo Custodero (Azienda Ospedaliero - Universitaria Consorziale Policlinico di Bari, Bari, Medicina Interna Universitaria C. Frugoni);

- Luigi Fenoglio, Andrea Falcetta (Azienda Sanitaria Ospedaliera Santa Croce e Carle di Cuneo, Cuneo, S. C. Medicina Interna);

- Anna L. Fracanzani, Silvia Tiraboschi, Annalisa Cespiati, Giovanna Oberti, Giordano Sigon
(Fondazione IRCCS Cà Granda Ospedale Maggiore Policlinico, Milano, Medicina Interna 1B);

- Flora Peyvandi, Raffaella Rossio, Giulia Colombo, Pasquale Agosti (Fondazione IRCCS Cà Granda Ospedale Maggiore Policlinico, Milano, UOC Medicina generale - Emostasi e trombosi);

- Valter Monzani, Valeria Savojardo, Giuliana Ceriani (Fondazione IRCCS Cà Granda Ospedale Maggiore Policlinico, Milano, Medicina Interna Alta Intensità);

- Francesco Salerno, Giada Pallini (IRCCS Policlinico San Donato e Università di Milano, San Donato Milanese, Medicina Interna);

- Fabrizio Montecucco, Luciano Ottonello, Lara Caserza, Giulia Vischi (IRCCS Ospedale Policlinico San Martino e Università di Genova, Genova, Medicina Interna 1);

- Nicola Lucio Liberato, Tiziana Tognin (ASST di Pavia, UOSD Medicina Interna, Ospedale di Casorate Primo, Pavia);

- Francesco Purrello, Antonino Di Pino, Salvatore Piro (Ospedale Garibaldi Nesima, Catania, Unità Operativa Complessa di Medicina Interna);

- Renzo Rozzini, Lina Falanga, Maria Stella Pisciotta, Francesco Baffa Bellucci, Stefano Buffelli (Ospedale Poliambulanza, Brescia, Medicina Interna e Geriatria);

- Giuseppe Montrucchio, Paolo Peasso, Edoardo Favale, Cesare Poletto, Carl Margaria, Maura Sanino (Dipartimento di Scienze Mediche, Università di Torino, Città della Scienza e della Salute, Torino, Medicina Interna 2 U. Indirizzo d'Urgenza);

- Francesco Violi, Ludovica Perri (Policlinico Umberto I, Roma, Prima Clinica Medica);

- Luigina Guasti, Luana Castiglioni, Andrea Maresca, Alessandro Squizzato, Leonardo Campiotti, Alessandra Grossi, Roberto Davide Diprizio (Università degli Studi dell'Insubria, Ospedale di Circolo e Fondazione Macchi, Varese, Medicina Interna I);

- Marco Bertolotti, Chiara Mussi, Giulia Lancellotti, Maria Vittoria Libbra, Matteo Galassi, Yasmine Grassi, Alessio Greco (Università di Modena e Reggio Emilia, Azienda Ospedaliero-Universitaria di Modena; Ospedale Civile di Baggiovara, Unità Operativa di Geriatria);

- Angela Sciacqua, Maria Perticone, Rosa Battaglia, Raffaele Maio (Università Magna Grecia Policlinico Mater Domini, Catanzaro, Unità Operativa Malattie Cardiovascolari Geriatriche); 
- Vincenzo Stanghellini, Eugenio Ruggeri, Sara del Vecchio (Dipartimento di Scienze Mediche e Chirurgiche, Unità Operativa di Medicina Interna, Università degli Studi di Bologna/Azienda Ospedaliero - Universitaria S.Orsola Malpighi, Bologna);

- Andrea Salvi, Roberto Leonardi, Giampaolo Damiani (Spedali Civili di Brescia, U.O. 3a Medicina Generale);

- William Capeci, Massimo Mattioli, Giuseppe Pio Martino, Lorenzo Biondi, Pietro Pettinari (Clinica Medica, Azienda Ospedaliera Universitaria - Ospedali Riuniti di Ancona);

- Riccardo Ghio, Anna Dal Col (Azienda Ospedaliera Università San Martino, Genova, Medicina III);

- Salvatore Minisola, Luciano Colangelo, Mirella Cilli, Giancarlo Labbadia (Policlinico Umberto I, Roma, SMSC03 - Medicina Interna A e Malattie Metaboliche dell'osso);

- Antonella Afeltra, Benedetta Marigliano, Maria Elena Pipita (Policlinico Campus Biomedico Roma, Roma, Medicina Clinica);

- Pietro Castellino, Luca Zanoli, Alfio Gennaro, Agostino Gaudio (Azienda Ospedaliera Universitaria Policlinico V. Emanuele, Catania, Dipartimento di Medicina);

- Valter Saracco, Marisa Fogliati, Carlo Bussolino (Ospedale Cardinal Massaia Asti, Medicina A);

- Francesca Mete, Miriam Gino (Ospedale degli Infermi di Rivoli, Torino, Medicina Interna)

- Carlo Vigorito, Antonio Cittadini (Azienda Policlinico Universitario Federico II di Napoli, Napoli, Medicina Interna e Riabilitazione Cardiologica);

- Guido Moreo, Silvia Prolo, Gloria Pina (Clinica San Carlo Casa di Cura Polispecialistica, Paderno Dugnano, Milano, Unità Operativa di Medicina Interna);

- Alberto Ballestrero, Fabio Ferrando, Roberta Gonella, Domenico Cerminara (Clinica Di Medicina Interna ad Indirizzo Oncologico, Azienda Ospedaliera Università San Martino di Genova);

- Sergio Berra, Simonetta Dassi, Maria Cristina Nava (Medicina Interna, Azienda Ospedaliera Guido Salvini, Garnagnate, Milano);

- Bruno Graziella, Stefano Baldassarre, Salvatore Fragapani, Gabriella Gruden (Medicina Interna III, Ospedale S. Giovanni Battista Molinette, Torino);

- Giorgio Galanti, Gabriele Mascherini, Cristian Petri, Laura Stefani (Agenzia di Medicina dello Sport, AOUC Careggi, Firenze);

- Margherita Girino, Valeria Piccinelli (Medicina Interna, Ospedale S. Spirito Casale Monferrato, Alessandria);
- Francesco Nasso, Vincenza Gioffrè, Maria Pasquale (Struttura Operativa Complessa di Medicina Interna, Ospedale Santa Maria degli Ungheresi, Reggio Calabria);

- Leonardo Sechi, Cristiana Catena, Gianluca Colussi, Alessandro Cavarape, Andea Da Porto (Clinica Medica, Azienda Ospedaliera Universitaria, Udine).

- Nicola Passariello, Luca Rinaldi (Presidio Medico di Marcianise, Napoli, Medicina Interna);

- Franco Berti, Giuseppe Famularo, Patrizia Tarsitani (Azienda Ospedaliera San Camillo Forlanini, Roma, Medicina Interna II);

- Roberto Castello, Michela Pasino (Ospedale Civile Maggiore Borgo Trento, Verona, Medicina Generale e Sezione di Decisione Clinica);

- Gian Paolo Ceda, Marcello Giuseppe Maggio, Simonetta Morganti, Andrea Artoni, Margherita Grossi (Azienda Ospedaliero Universitaria di Parma, U.O.C Clinica Geriatrica);

- Stefano Del Giacco, Davide Firinu, Giulia Costanzo, Giacomo Argiolas (Policlinico Universitario Duilio Casula, Azienda Ospedaliero-Universitaria di Cagliari, Cagliari, Medicina Interna, Allergologia ed Immunologia Clinica);

- Giuseppe Montalto, Anna Licata, Filippo Alessandro Montalto (Azienda Ospedaliera Universitaria Policlinico Paolo Giaccone, Palermo, UOC di Medicina Interna);

- Francesco Corica, Giorgio Basile, Antonino Catalano, Federica Bellone, Concetto Principato (Azienda Ospedaliera Universitaria Policlinico G. Martino, Messina, Unità Operativa di Geriatria);

- Lorenzo Malatino, Benedetta Stancanelli, Valentina Terranova, Salvatore Di Marca, Rosario Di Quattro, Lara La Malfa, Rossella Caruso (Azienda Ospedaliera per l'Emergenza Cannizzaro, Catania, Clinica Medica Università di Catania);

- Patrizia Mecocci, Carmelinda Ruggiero, Virginia Boccardi (Università degli Studi di Perugia-Azienda Ospedaliera S.M. della Misericordia, Perugia, Struttura Complessa di Geriatria);

- Tiziana Meschi, Andrea Ticinesi, Antonio Nouvenne (Azienda Ospedaliera Universitaria di Parma, U.O Medicina Interna e Lungodegenza Critica);

- Pietro Minuz, Luigi Fondrieschi, Giandomenico Nigro Imperiale (Azienda Ospedaliera Universitaria Verona, Policlinico GB Rossi, Verona, Medicina Generale per lo Studio ed il Trattamento dell'Ipertensione Arteriosa); 
- Mario Pirisi, Gian Paolo Fra, Daniele Sola, Mattia Bellan (Azienda Ospedaliera Universitaria Maggiore della Carità, Medicina Interna 1);

- Massimo Porta, Piero Riva (Azienda Ospedaliera Universitaria Città della Salute e della Scienza di Torino, Medicina Interna 1U);

- Roberto Quadri, Erica Larovere, Marco Novelli (Ospedale di Ciriè, ASL TO4, Torino, S.C. Medicina Interna);

- Giorgio Scanzi, Caterina Mengoli, Stella Provini, Laura Ricevuti (ASST Lodi, Presidio di Codogno, Milano, Medicina);

- Emilio Simeone, Rosa Scurti, Fabio Tolloso (Ospedale Spirito Santo di Pescara, Geriatria);

- Roberto Tarquini, Alice Valoriani, Silvia Dolenti, Giulia Vannini (Ospedale San Giuseppe, Empoli, USL Toscana Centro, Firenze, Medicina Interna I);

- Riccardo Volpi, Pietro Bocchi, Alessandro Vignali (Azienda Ospedaliera Universitaria di Parma, Clinica e Terapia Medica);

- Sergio Harari, Chiara Lonati, Federico Napoli, Italia Aiello (Ospedale San Giuseppe Multimedica Spa, U. O. Medicina Generale);

- Raffaele Landolfi, Massimo Montalto, Antonio Mirijello (Policlinico Universitario A. Gemelli Roma, Clinica Medica);

- Francesco Purrello, Antonino Di Pino (Ospedale Garibaldi - Nesima - Catania, U.O.C Medicina Interna);

- Nome e Cognome del Primario, Silvia Ghidoni (Azienda Ospedaliera Papa Giovanni XXIII, Bergamo, Medicina I);

- Teresa Salvatore, Lucio Monaco, Carmen Ricozzi (Policlinico Università della Campania L. Vanvitelli, UOC Medicina Interna);

- Alberto Pilotto, Ilaria Indiano, Federica Gandolfo (Ente Ospedaliero Ospedali Galliera Genova, SC Geriatria Dipartimento Cure Geriatriche, Ortogeriatria e Riabilitazione)

\section{Funding}

The work was partly funded by the MIUR-Italy grant "Dipartimenti di Eccellenza 2018-2022" assigned to the Department of Biomedical, Metabolic and Neural Sciences of the University of Modena and Reggio Emilia.

\section{Disclosure}

The authors report no conflicts of interest in this work.

\section{References}

1. United Nations.World Population Ageing 2019. Available from: https:// www.un.org/en/development/desa/population/publications/pdf/ageing/ WorldPopulationAgeing2019-Highlights.pdf. December 20, 2020.

2. Long SB, Blaha MJ, Blumenthal RS, Michos ED. Clinical utility of rosuvastatin and other statins for cardiovascular risk reduction among the elderly. Clin Interv Aging. 2011;6:27-35.

3. Bertolotti M, Mussi C, Pellegrini E, et al. Age-associated alterations in cholesterol homeostasis: evidence from a cross-sectional study in a Northern Italy population. Clin Interv Aging. 2014;9:425-432.

4. Mach F, Baigent C, Catapano AL, et al. 2019 ESC/EAS Guidelines for the management of dyslipidaemias: lipid modification to reduce cardiovascular risk. Eur Heart J. 2020;41(1):111-188.

5. Bibbins-Domingo K, Grossman DC, Curry SJ, et al. Statin use for the primary prevention of cardiovascular disease in adults: US Preventive Services Task Force Recommendation Statement. JAMA. 2016;316:1997-2007.

6. Singh S, Zieman S, Go AS, et al. Statins for primary prevention in older adults-moving toward evidence-based decision-making. $J \mathrm{Am}$ Geriatr Soc. 2018;66(11):2188-2196.

7. Verdoia M, Galasso G, Filardi PP, De Luca G. Statins and elderly: from clinical trials to daily practice. Curr Vasc Pharmacol. 2019;17 (3):233-238.

8. Grundy SM, Stone NJ, Bailey AL, et al. 2018 AHA/ACC/AACVPR/ AAPA/ABC/ACPM/ADA/AGS/APhA/ASPC/NLA/PCNA guideline on the management of blood cholesterol. $\mathrm{J}$ Am Coll Cardiol. 2019;73(24):e285-e350.

9. Gazzola K, Vigna GB. Hypolipidemic drugs in elderly subjects: indications and limits. Nutri Metabol e Cardiovasc Dis. 2016;26:1064-1070.

10. Miller MD, Towers A. Manual of Guidelines for Scoring the Cumulative Illness Rating Scale for Geriatrics (CIRS-G). Pittsburg: University of Pittsburgh; 1991:596.

11. Mahoney FI, Barthel DW. Functional evaluation: the Barthel index. Md State Med J. 1965;14:61-65.

12. Katzman R, Brown T, Fuld $\mathrm{P}$, et al. Validation of a short orientation-memory-concentration test of cognitive impairment. Am J Psychiatry. 1983;140:734-739.

13. Ardoino I, Franchi C, Nobili A, et al. Pain and frailty in hospitalized older adults. Pain Ther. 2020;9:727-740.

14. Franchi C, Mannucci P, Nobili A, Ardoino I, REPOSI investigators. Use and prescription appropriateness of drugs for peptic ulcer and gastrooesophageal reflux disease in hospitalized older people. Eur J Clin Pharmacol. 2020;76:459-465.

15. European Medicines Agency. Guideline for Good Clinical Practice E6 (R2). London: European Medicines Agency; 2016. Available at: https:// www.ema.europa.eu/en/documents/scientific-guideline/ich-e-6-r2guideline-good-clinical-practice-step-5_en.pdf. Accessed June 9, 2021.

16. Marcucci M, Franchi C, Nobili A, et al. Defining aging phenotypes and related outcomes: clues to recognize frailty in hospitalized older patients. J Gerontol a Biol Sci Med Sci. 2017;72(3):395-402.

17. Greenacre MJ. Correspondence Analysis in Practice. London, UK: Academic; 1993:32-37.

18. Husson F, Le S, Pages J. Exploratory Multivariate Analysis by Example Using R. Boca Raton, FL: Chapman \& Hall/CRC; 2011.

19. Hennig C. Cluster wise assessment of cluster stability. Comput Stat Data Anal. 2007;52:258-271.

20. De La Higuera L, Riva E, Djade CD, et al. Prognostic value of estimated glomerular filtration rate in hospitalized elderly patients. Intern Emerg Med. 2014;9:735-747.

21. Damiani I, Corsini A, Bellosta S. Potential statin drug interactions in elderly patients: a review. Expert Opin Drug Metab Toxicol. 2020;16 (12):1133-1145. doi:10.1080/17425255.2020.1822324 
22. De Pádua Borges R, Degobi NAH, Bertoluci MC. Choosing statins: a review to guide clinical practice. Arch Endocrinol Metab. 2020;64 (6):639-653. doi:10.20945/2359-3997000000306

23. Toth PP, Patti AM, Giglio RV, et al. Management of Statin Intolerance in 2018: still more questions than answers. $\mathrm{Am}$ J Cardiovasc Drugs. 2018;18(3):157-173. doi:10.1007/s40256-0170259-7

24. Tibuakuu M, Blumenthal RS, Martin SS Bempedoic Acid for LDL-C Lowering: what Do We Know? Available from: https:// www.acc.org/latest-in-cardiology/articles/2020/08/10/08/21/bempe doic-acid-for-ldl-c-lowering\#: : text=Bempedoic $\% 20 \mathrm{acid} \% 20 \mathrm{is} \% 20 \mathrm{a}$ $\% 20$ new,biosynthesis $\% 20$ pathway $\% 20$ in $\% 20$ the $\% 20$ liver.\&text= Whereas $\% 20$ statins $\% 20$ inhibit $\% 20 \mathrm{HMG} \% 20 \mathrm{CoA}$, upstream $\% 20$ of $\%$ $20 \mathrm{HMG} \% 20 \mathrm{CoA} \% 20$ reductase.

25. The Medicines Utilisation Monitoring Centre. National Report on Medicines use in Italy 2019. Rome: Italian Medicines Agency, 2020.

26. Viscogliosi G, Donfrancesco C, Lo Noce C, et al. Prevalence and correlates of statin underuse for secondary prevention of cardiovascular disease in older adults 65-79 years of age: the Italian health examination survey 2008-2012. Rejuvenation Res. 2020;23 (5):394-400. doi:10.1089/rej.2019.2268

27. Arca M, Ansell D, Averna M, et al. Statin utilization and lipid goal attainment in high or very-high cardiovascular risk patients: insights from Italian general practice. Atherosclerosis. 2018;271:120-127. doi:10.1016/j.atherosclerosis.2018.02.024

28. Liguori I, Aran L, Bulli G, et al. Statins in cardiovascular prevention in the oldest-old. A black hole. $J$ Gerontol Geriatrics. 2017;65:263-270.
29. Cholesterol Treatment Trialists' Collaboration. Efficacy and safety of statin therapy in older people: a meta-analysis of individual participant data from 28 randomised controlled trials. The Lancet. 2019;393:407-415.

30. Ruscica M, Macchi C, Pavanello C, Corsini A, Sahebkar A, Sirtori CR. Appropriateness of statin prescription in the elderly. Eur J Intern Med. 2018;50:33-40.

31. Bertolotti M, Lancellotti G, Mussi C. Management of high cholesterol levels in older people. Geriatr Gerontol Int. 2019;19 (5):375-383.

32. Stone NJ, Robinson JG, Lichtenstein AH, et al. ACC/AHA guideline on the treatment of blood cholesterol to reduce atherosclerotic cardiovascular risk in adults: a report of the American College of Cardiology/American Heart Association task force on practice guidelines. J Am Coll Cardiol. 2013;2014(63):2889-2934.

33. Borne E, Meyer N, Rybarczyck-Vigouret MC, et al. Potential statin overuse in older patients: a retrospective cross-sectional study using French health insurance databases. Drugs Aging. 2019;36:947-955.

34. Bertolotti M, Franchi C, Rocchi MBL, et al.; On behalf of REPOSI Investigators. Prevalence and determinants of the use of lipidlowering agents in a population of older hospitalized patients: the findings from the REPOSI (REgistro POliterapie Società Italiana di Medicina Interna) Study. Drugs Aging. 2017;34:311-319.
Clinical Interventions in Aging

\section{Publish your work in this journal}

Clinical Interventions in Aging is an international, peer-reviewed journal focusing on evidence-based reports on the value or lack thereof of treatments intended to prevent or delay the onset of maladaptive correlates of aging in human beings. This journal is indexed on PubMed Central, MedLine, CAS, Scopus and the Elsevier

\section{Dovepress}

Bibliographic databases. The manuscript management system is completely online and includes a very quick and fair peer-review system, which is all easy to use. Visit http://www.dovepress.com/ testimonials.php to read real quotes from published authors. 